Supposons, par exemple, que $\frac{\mathrm{L}_{1}}{a_{1}}=\frac{\mathrm{L}_{2}}{a_{3}}$ et reprenons les valeurs que nous avions au bableau du chapitre premier, Irailant ce cus spécial.

Si : $\frac{a_{2}}{n_{1}}=0,82$ el $D_{1} / D_{2}=0,7, \cos m \frac{L_{1}}{a_{1}}=0,536$ dans le cas d'unc seule conduite. Dans le cas de deux conduites, on aurail $\frac{\mu^{\prime}}{2}$ an lieu de $\mu^{\prime}$, ce qui revient à prendre $\frac{\left(D_{1}+D_{2}\right)}{D_{3}}$ $=1,4$ et $\cos m^{\prime} \frac{L_{1}}{a_{1}}=0,785$. Le fait d'avoir deux conduites jumeléos augmente sensiblement les surcharges le long de l'ensemble de la conduite.

\section{III. - CONCLUSIONS}

Qu'on nousı permette de faire ici quelques remarques et d'en souligner l'importance. Nous avons exposé dans notre "Théorie Générale " une méthode de calcul des conduites forcécs et des chambres d'équilibre, supposant une fermelure linéaire plus ou moins lente des organes d'obturation. Nous voici incités à proposer, pour les conduites et les chambres, un calcul de contrôle que nous justifierons par les considémations suivantes :

Tout coup de bélier de fermeture totale engendre, une fois la fermeture achevée, un mouvement périodique qui ne s'amortit que très lentement. Ainsi que nous l'avons vu plus haut, ce mouvement intéresse l'ensemble du système complexe : conduite forcée, chambre d'équilibre, galerie de mise en charge. Supposons que, lors d'un mouvement de fermeture linéaire lente, la surcharge devant l'obturateur $O$ soil $B_{\mathrm{m}}$ (environ 10 à $25 \%$ de $y_{0}$ ). On aura la valeur du coup de béflicr au poinl $\mathrm{A}$, point de discontinuité - en admeltant qu'un mouvement périodique s'est établi - en substituant dans (10) B charge au point $\mathrm{A}$ est :

$$
\dot{\mathrm{B}}_{\mathrm{m}}=\mathrm{B}_{\mathrm{m}} \cos m \frac{\mathrm{L}_{1}}{a_{1}}=\mathrm{B}_{\mathrm{m}} \sin \frac{\pi}{2} \frac{E}{\mathrm{I}}
$$

On vérifiera que, dans certains cas, cetle valeur de contrôle peul donner des valeurs supérieures à la valeur calculéc d'après les formules de notre "Théorie générale ".

Il y a lieu, enfin, d'examiner, comme cas extrême, la possibilité d'un mouvement de résonance. En ce cas, la surcharge au bas de la chambre d'équilibre est donnée directement par la formule (10), où l'on pose $\mathrm{A}=y_{0}$, et les valeurs numériques que l'on obtiendra seront de beaucoup plus élevées que celles données par l'une ou l'autre méthode admettant une fermeture lente. Il est vraisemblable qu'en cas de résonance, la chambre d'équilibre constituera un point faible du système.

Ainsi que nous l'avons rappelé au cours de celle note, on connaît un certain nombre de mesures qui confirment les calculs eflectués par nous. Mais il importe de reprendre ces mesures et vérifications sur une plus large échelle. Ce n'est qu'après avoir achevé ces vérifications que l'on pourra conclure et aborder utilement l'étude de l'amortissement des oscillations périodiques (24).

Deux nouveaux chapitres pourront alors être mis à l'étude : la superposition des ondes de coup de bélier aux oscillations en masse dans les chambres d'équilibre et l'analyse de la résonance du fondamental dans les systèmes de conduites complexes.

\section{Ch. JAEGER.}

(24) Pour le cas des conduites simples on lira avec fruit l'étude de LE Conte : Reprinted papers an of the A.S.M.E. University of California 1934.

\title{
La Houille Blanche devrait concourir à la production des carburants nationaux en France
}

\author{
par V. Charrin, Ingénieur-Conseil
}

A un certain moment, on avait eu de sérieuses craintes de voir la houille blanche porter atteinte à notre industrie houillère et amener son déclin avant l'épuisement des gìtes de charbon. Hleureusement, on voyait mal, et malgré le beau développement pris chez nous par l'énergie hydraulique, malgré même une nouvelle source d'énergie dans laquelle on ne comptait guère, il y a trente ans : le pétrole, la consommation de charbon s'est maintenue à un taux qu'on peut considérer normal.

La crise dont souffre depuis quatre à cinq ans nos bassins houillers et le chômage qui en résulte, ne sont pas dûs à la concurrence des autres modes de production de l'énergie, ils découlent surtoul des importations que nous continuons, peu judicieusement d'ailleurs, à demander à l'étranger. Qu'on se contenle de recevoir du dehors, les quantités et les qualités que nous ne pouvons pas produire nous-même et de suile, nos houillères connaîtront une c̀re de prospérité en même temps que nos prix de revient iront enfin à la baisse.

Les chiffres se rapportant, pour le monde entier, aux trois sources principales d'énergie sont caractérisțiques. En prenant ceux de l'année d'avant-guerre et ceux de 1929 - à partir de cette date les chiffres sont affectés par la crise actuelle - , on voit que la production de houille est passée de 1340 à 1538 millions de tonnes ; celle de l'énergie hydroélectrique, de 50 à 120 milliards de kilowatts-heure et celle du pétrole brut, de 55 à 212 millions de tonnes.

On voit nettement que malgré des multiples de l'ordre de 2 et de 4, pour la houille blanche et pour les hydrocarbures liquides, la production du charbon a continué une marche ascendiante.

En France, présentement, sur les 15 à 16 milliards de KWH consommés annuellement (les installations totales comportent 19 milliards de KWH, soit un suréquipement de 3 à ' milliards) l'énergie thermique participe environ pour la moitié.

L'évolution semble donc tout à fait normale et si la houille noire, forcément limitée dans ses réserves, doit faire place un jour à d'autres sources d'énergie, elle le fait progressivement et tout porte à croire qu'elle jouera encore un rôle important pendant longtemps.

Mieux que cela, devant la demande sans cesse croissante 
d'hydrocarbures liquides et devant les réserves relativement faibles en produits naturels de toute la planète, ne va-l-on pas voir la houille blanche prêter son concours à la houille noire pour obtenir artificiellement ces hydrocarbures liquides. Toutes ces sources d'énergie ne seront donc plus des concurrentes comme on voulait le dire, mais bel et bien des collaboratrices.

En effet, déjà de nombreux pays ont abordé le problème de l'obtention des carburants en partant des hydrocarbures solides (houilles, lignites, pyroschistes, tourbes, etc.) ; les uns le font dans le but de conserver leur indépendance; les autres pour avoir des produits moins coûteux ; d'autres, enfin, en prévision de l'épuisement des sources naturelles de pétrole.

Ne voit-on pas les Etats-Unis d'Amérique, pays du naphte cependant, envisager l'utilisation prochaine de leurs immenses réserves de schistes bitumeux, déjà évaluées à 108 milliards de lbarils - soit 17 milliards de tonnes de pétrole brut. - C'est ce qui ressort nettement de la dernière assemblée générale annuelle de l'Américan Petroleum Institute.

En France, le problème de l'alimentation en carburants se pose plus aigu que dans beaucoup d'autres pays, v'u la pauvreté à peu près certaine du sous-sol et la consommation qui croît constamment. Pour l'an dernier, nos besoins ont dépassé 6 millions et demi de tonnes de produits pétrolifères et notre production s'en est tenue à 300.000 tonnes environ, en y comprennant Péchelbronn, Autun, Gabian, les benzols et les alcools.

On sait que les Allemands, placés dans une situation identique à la nòtre, ont résolu la question par l'obtention de carburants synthétiques à partir des combustibles solides et principalement des lignites. A la suite de recherches, nuxquelles nombre de savants et inventeurs français se sont intéressés, on a reconnu que lie seul moyen réellement industriel pour arriver aux carburants de synthèse à un prix convenable, résidait dans l'hydrogénation des huiles brutes provenant de carbonisation de houilles et surtout des lignites et des pyroschistes.

L'opération comprend donc deux phases successives, mais bien distinctes l'une de l'autre ; premièrement, la carbonisation de la roche hydrocarburée - on désigne improprement cette phase sous le nom de distillation - et, deuxièmement, l'addition, sous conditions déterminées, d'hydrogène aux huiles ainsi produites, pour arriver le plus près possible de la saturation.

La première phase s'effectue par les propres moyens de la s'ubstance mise en œuvre; une partie des calories qu'elle renferme étant employés à la transformation du restant : mais, dans la seconde, il faut obligatoirement un apport d'hydrogène et c'est là où nous envisageons le concours de la houille blanche.

Jusqu'à maintenant, le coût élevé de l'hydrogène a été considéré comme le plus grand obstacle ì l'obtention pratique des carburants de synthèse en partant des combustibles solides. On évalue, dans les oas les plus propices, à 900 mètres cubes, la quantité d'oxygène utile à la production d'une tonne d'essence. Quand on hydrogène directement le charbon, il en faut 2 à 3 fois plus et c'est pour cela qu'on abandonne presque complètement ce mode de traitement, on se rend compte aussitòt, en appliquant à l'hydrogène le prix actuel de 25 à 30 centimes le mètre cube, de combien il grève le coût de l'essence finale. Il y a donc nécessité, en première ligne, d'abaisser le prix de revient du gaz hydrogène. C'est là que chez nous, la houille blanche a un rôle tout indiqué.

Les moyens d'obtenir l'hydrogène sont nombreux, nous n'en voulons pas faire l'énumération ; ceux qui pc'uvent frecevoir des applinations industrielles exigent des consommations élevés d'énergie qu'on ne peut songer à demander qu'à la houille blanche.

La région en France qui est privilégiée pour renfermer des lignites extrêmement propres ì la fabrication des carburanls, correspond sensiblement au bassin idu Rhône ; c'est là que gisent les lignites de Fuveau, de Manosque, de Bagnols-surCèze, de la Tour-du-Pin, de Soblay, etc., etć. (on pourrail y ajouter des pyroschistes de Vagnas, de Boson, des Causses, du Jura, etc.) dont la plupart sont inutilisés aujourd'hui. Or, on parle de plus en plus de l'aménagement du Rhône au double point de vue navigation et force motrice; la logique exige que celle-ci soit utiliséc à mettre en valeur les ressources régionales, dont les lignites, comme nous venons de le dire, font partie.

Nous savons, d'autre part, que le ministre des Travaux publics vient de mettre à l'enquête le projel du grand barrage de Génissiat (Ain). On se souvient que ce projet, qui date de près de 30 ans, comporte dans les Gorges du Rhône, entre Seyssel et Bellegarde, un barrage de 80 mètres de hauteur environ qui transformera la vallée en un lac de 23 kilomètres de longueur, quoique ne couvrant que 380 hectares. Il en résultera non seulement la navigalion possible entre Genissiat et P'ougny, près de Genève, mais également une source d'énergie évaluée à 59.000 Kilowatts au moment de l'étiage et à 200.000 et plus lors des hautes eaux.

Ce premier aménagement du Rhône peut donc être le point de départ de l'hydrogène bon marché et par suite de l'obtenlion à bon compte des carblurants synthétiques, qui, cux, seront cent pour cent nationaux, puisqu'ils proviendront des lignites que nous avons méprisés à peu près totalemenl jusqu'aujourd'hui.

La houille blanche a permis il y a cinquante ans, à l'aluminium de devenir un métal d'usage contrant, elle permettra demain à nos inépuisables réserves de roches pétroligènes de se transformer pratiquement en essence.

\section{Expériences sur les piles de barrages déversoirs à profil aérodynamique et à contractions nulles}

Note ${ }^{(1)}$ de MM. Léopold Escande et Georges Sabathé.

L'un de nous $\left(^{2}\right)$ avait étudié le profil optimum de barrage déversoir dont il avait indiqué la détermination pratique. Dans ce même travail, il avait proposé inne méthode pour la détermination de piles à profil aérodynamique susceptibles de présenter un net avantage vis-à-vis des profils usuels.
La méthode est la suivante : on trace, dans un plan, un profil d'aile biconvexe symétrique ; on déforme ensuite ce plan, en l'appliquant sur la surface cylindrique constiluant le déversoir, de telle sorte que le profil, d'axe normal aux génératrices du barrage, ait son maître couple au voisinage 